\title{
Identifying and Tracking the Object Motion Path By Means Of Image Processing In Python through the CanopenProtocol
}

\author{
Farzaneh Goli 1,*, Vahid Noei 2. \\ ${ }^{1}$ Mechatronics Engineering Department, South Tehran Branch, \\ Islamic Azad University, Tehran, Iran. \\ st_f_goli@azad.ac.ir \\ ${ }^{2}$ Mechatronics Engineering Department, South Tehran Branch, \\ Islamic Azad University, Tehran, Iran. \\ st_v_noei@azad.ac.ir
}

\begin{abstract}
In the field of machine vision, object tracking is one of the crucial topics. Tracking is the positioning process of moving targets by means of a camera. Nowadays, image processing systems are used in different usages. In the current research, at first, the object will be selected by image processing in Python with opencv and color detection. For better control, then the necessary calculations will bedonein the programmable logic controller (plc), and finally, through the control area network (CAN) protocol, the object's position will be transferred to two servomotors in both $X$ and $Y$ directions. Due to the fact that the engines velocity will be proportional to the object movement speed, the selected object will be traced in real time.
\end{abstract}

Keywords: Object Tracking, CAN protocol, Python, Opencv

\section{Introduction}

Objecttracking is one of the most significant and useful branches in various fields, such as surveillance, aerospace and autonomous navigation. Nonetheless, tracking several objects in real time is often a very challenging task. Morimitsua et al.(2016) haveoffered a new approach to deploy structural relationships in order to track numerousobjectsso that in the first frame of the video, ittracks all objects online. In this project, the aim isobject tracking and another object movementin coordination with a moving object.

The fixed base industrial robots have multipurpose control and programmingcapabilities, and they are used to move material, components, tools, or specialized devices through planned movements. Industrial robots have been substituted by humans in order to accomplishvarious kinds of tasks, including dangerous, uniform or severe work that humans are not able to do. These activities are regularly carried out in low-ventilated spaces or filled with toxic gases. Industrial robots are progressing to be smart and automated.

Over the past few years, path tracking control in industrial robots has attracted much attentionas an essential and critical issue. There are numerous types of methods to control the precise paths tracking in industrial robots. Due to their simple structure and controlling the designedautonomous model,proportional integral derivatives(PIDs) areextensively used to control the movement of robots (Ayala et al., 2012).

Though, PID controllers should be adjusted according to different operating conditions, which are typically time-consuming. Target tracking control algorithm in a group of mobile robots by camera tracking is proposed in (Wang and $\mathrm{Gu}, 2012$ ). A distributed Kalman filter is also designed to approximation the target location. The effectiveness of the control algorithm is confirmed by simulation and empirical results. Anadaptationpathtracking controller (Mohareri et al., 2012) is designed to track the motion of a robot with unidentified parameters and unknown dynamics. By means of neural networks learning ability,the Back-step controller is designed to preventdynamics recognition of robot. To investigate the performance of the designed controller algorithm with a Back-step controller on a platform the commercial robot was simulated and tested.

For target prediction controlling,in Li et al.(2016), a planwas presented, which includes dynamic neural optimization for target movement path tracking. Target prediction has been controlled by means of repetitive quadratic planning methods. Then, in order to solve a problem in a lagged horizon, aninitial neural network was used that the validation of the control method has been confirmed based on the experiments.

A proportional derivative(PD) controller is offered with sliding mode control theory for robotic tracking controlling in (Ouyang et al, 2014). In the control theory, the simple linear control features are provided by means of PD control and non-linear control through sliding control. Yet, for the validation and theoretical strength, only the simulation results of control were presented. 
A reversible adaptive control plan (Hu et al., 2012) has been suggested to trace motion paths of robot arms in the presence of external disturbances and variable parameters. The controller was converted to a robotic system by means of an adaptive technique to evaluate the uncertainty of the robotics system. The effectiveness of the controllers is approvedjust by numerical simulation results.

A sliding controller with constant speed in (Corradini et al., 2012) is designed from robot arms connected to an uncertainty estimator for path tracking. Path tracking function was evaluated based on experimental results.

The CAN communication protocol was first designed by Bush for cars and was released in the late 80's and used by European and then American automakers. The protocol was recognized by the International Standards Organization under ISO 11898 ISO in 1991, and then it was developed in industrial automation, the auto industryand road transport, agricultural machinery and road construction. A version of that developed in industrial automation is now known as CANOpen and has found a special position in industrial automation. This network consists of a differential serial bus that connects different parts of the system (Rezaei,2016).

Actually, the CAN-based network is a serial and two-wire communication based on the 485 RS-standard, which links up to 321 sensors and different devices. This network worksbased on sending and receiving messages so that messages are flowed by sensors and control devices in the network and the receivers perform the operation by receiving a message related to the operation itself. Substantialreduction in wiring, flexibility, high reliability, messages prioritizing and proper troubleshooting features are among the benefits of this protocol. Information transmission in this protocol is done by a pair of wires and differentially, which minimizes the effect of noise. The length of the network can up to 3111 meters and the data transfer speed in this protocol can be up to $1 \mathrm{Mbps}$ (at a distance of $50 \mathrm{~m}$ ) (Rezaei, 2016).

\section{Method}

At first, in the current research, the object has been selected by means image processing in the Python program with the opencv library and placed in the system memory and by displacing the object in axes $\mathrm{X}$ and $\mathrm{Y}$, the amount of displacement is computed and extracted, to perform calculations and transferring the command to the servomotors the PLC is used (Fig. 1 and 2).

In order totransfer information for servomotor displacement,the CAN method has been used. Due to the lower number of wires and the higher data transfer speeds this method has been chosen.

\section{The sent frames in the CAN protocol}

I order that CAN network operate in real time and data tobe sent at the correct time, there are restrictionsin line with sending data packets (frames); so that each data packet cannot carry more than 8 bytes of data (Figure 3). Before explaining the data frames, it is necessary to mention that two different versions of the CAN standard are common today:

\section{-CAN 2.0A standard \\ -CAN 2.0B standard}

The difference between these two standards is in the number of identifier bitsso that in the CAN 2.0A standard, the acceptable number of identifier bits is 11 bits, and in the CAN 2.0B standard, the number of allowablebits is expanded up to 29 bits. Fortunately, the variations to these two standards are such that both standards can exchange data on a data line without causing interruptions (Figure 4).

As can be seen in Figure 4, each data packet has a unique address that depending on the CAN standard can have 11 or 29 bits. The number of data in each frame is determined by the DLC, that in the maximum state it can have 8 bytes. The next section is the CRC that it is produced and reviewed by hardware. If the address and CRC are correct, by sending ACK,informs the sender of receiving information.

In order to test the output and test results, the motors are installed on a table with two directions of freedomby means of Scroll method, and, according to the position that the PLC sends to motor drives, the table will be replaced. A pen is embedded on a table that according to the table movement in the $\mathrm{X}$ and $\mathrm{Y}$ directions, the pen draws a path on a white paper, in Figure 5, you can see the movement of the object and pen in 5 stages.

In order that the speed of the movement to besynchronicwith the object's displacement, the PID function,and its computational methods are performed based on the derivative and integral coefficients in the servomotor and the PLC drive (winprolader program). The advantage of this design in tracking is the speed of the reaction of the system to move the desired object.

\section{Findings}

In the present research, an object was trackedby means ofimage processing with Python, and the object's position was transmitted through the CAN protocol to two servomotors, and these two motors were installed on a table with a pen, and according to the object movement, servomotors, the table was moved and by means of the pen, itdraws the path. 
ISSN (Print) : 2319-8613

\section{Figures}

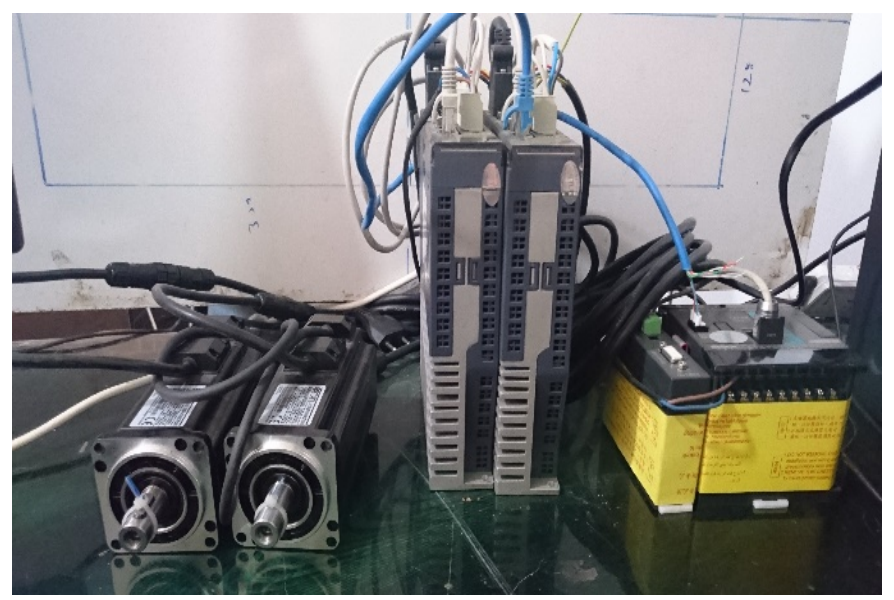

Figure 1: Information Transfer System to Servomotor

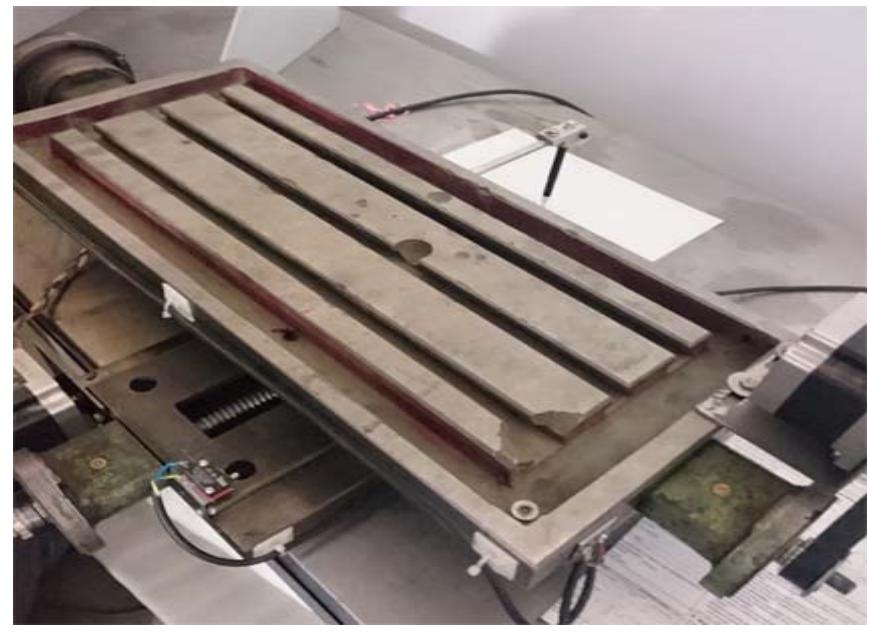

Figure 2: Two-degree freedom (2DOF) table equipped with a pen

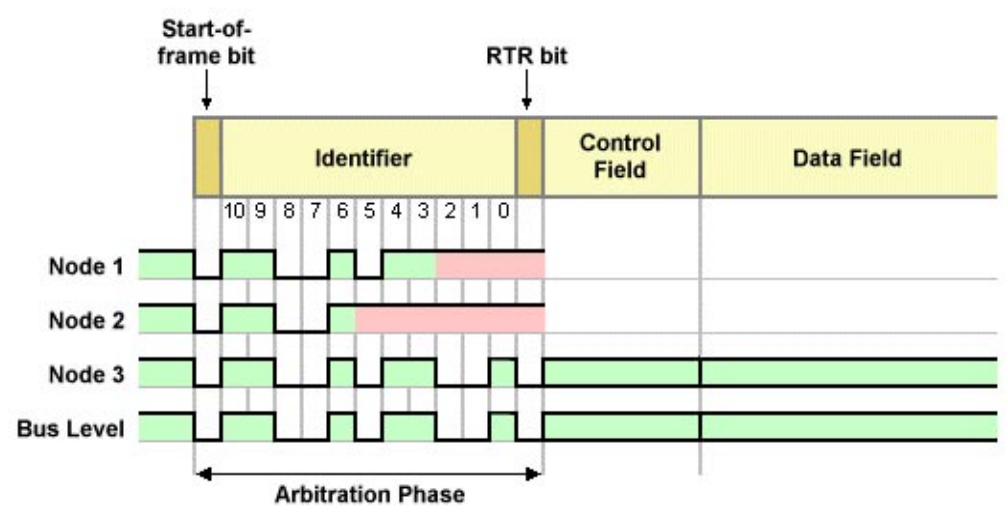

Figure 3:Sent forms in the CAN protocol 
CAN 2.0A (Standard Frame)

\begin{tabular}{l|c|c|c|c|c|c|c|c|c|c|} 
Frame & START & IDENTIFIER & RTR & IDE & rO & DLC & DATA & CRC & ACK & $\begin{array}{l}\text { EOF } \\
\text { IF }\end{array}$ \\
\hline
\end{tabular}

CAN 2.0B (Extended-Frame)

\begin{tabular}{|c|c|c|c|c|c|c|c|c|c|c|c|}
\hline START & IDENTIFIER & SRR & IDE & RTR & $r 1$ & ro & DLC & DATA & CRC & ACK & $\begin{array}{l}\text { EOF } \\
\text { IF }\end{array}$ \\
\hline 1 & 28 & 1 & 1 & 1 & 1 & 1 & 4 & $0 . .8$ & 15 & 2 & 10 \\
\hline
\end{tabular}

Figure 4: Differences between two different versions from the CAN standard

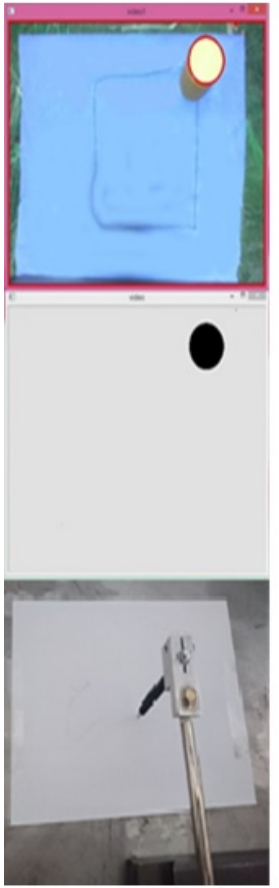

(1)

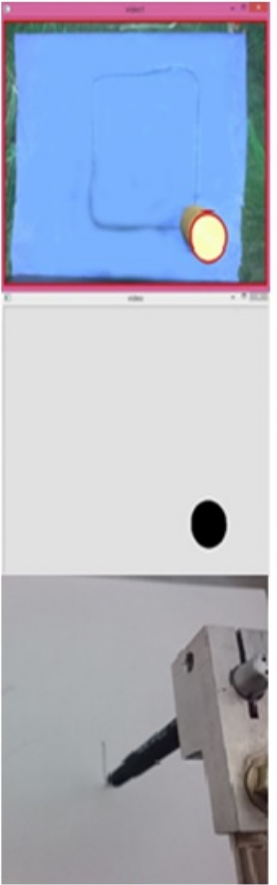

(2)

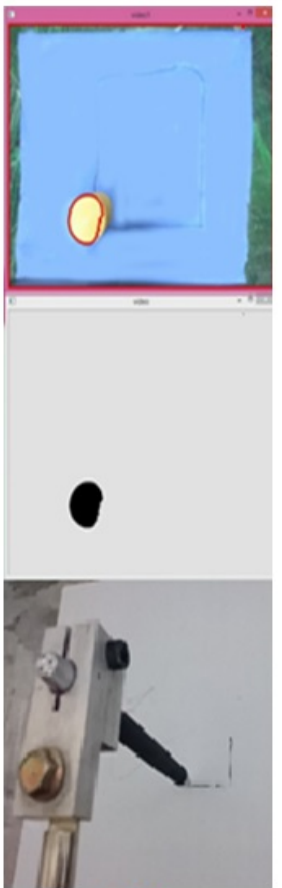

(3)

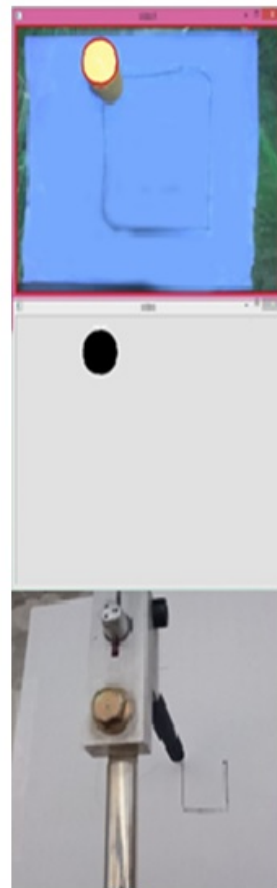

(4)

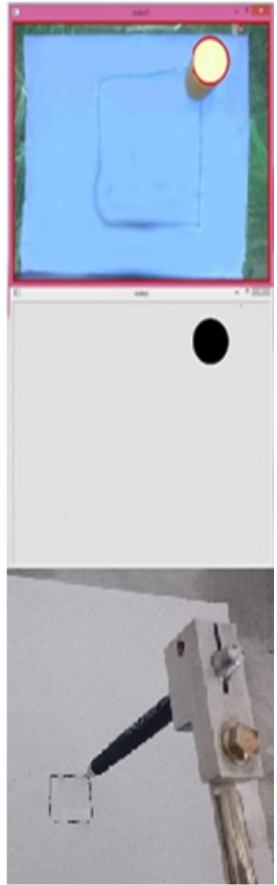

(5)

Figure 5: Comparison of Software and Hardware Output

\section{Discussion and Conclusion}

In the current research, the objects were tracked in Python by means of the opencv library. Then, the required calculations were done to control servomotors motion in plc, and finally, the object's position was transmitted via the CAN protocol to two servomotors in both $\mathrm{X}$ and $\mathrm{Y}$ directions. Due to the fact that the engine speed was proportional to the speed of the object movement, the selected object was trackedin real time.

\section{References}

[1] Rezaei, Amir, (2016). Introducing the CANopen Protocol and setting up the CBCAN-FBs F Module, Dorna Mehr SanaatCompany.

[2] H. Morimitsu, I. Bloch, R. M. Cesar-Jr. (2016).Exploring structure for long-term tracking of multiple objects in sports videos. Computer Vision and Image Understanding 000(2016). 1077-3142

[3] H.V.H. Ayala , L. dos Santos Coelho .(2012) Tuning of PID controller based on a multi-objec- tive genetic algorithm applied to a robotic manipulator, Expert Syst. Appl. 39 (10) (2012) 8968-8974 .

[4] Z. Wang, D. Gu. (2012). Cooperative target tracking control of multiple robots, IEEE Trans. Ind. Electron. 59 (8) (2012) 3232-3240 .

[5] O. Mohareri, R. Dhaouadi, A.B. Rad. (2012). Indirect adaptive tracking control of a nonholo- nomic mobile robot via neural networks, Neurocomputing 88 (2012) 54-66.

[6] Z. Li, J. Deng, R. Lu, et al. (2016). Trajectory-tracking control of mobile robot systems in- corporating neural-dynamic optimized model predictive approach, IEEE Trans. Syst. Man Cybern. 46 (6) (2016) 740-749.

[7] P.R. Ouyang, J. Acob, V. Pano. (2014). PD with sliding mode control for trajectory tracking of robotic system, Rob. Comput. Integr. Manuf. 30 (2) (2014) 189-200.

[8] Q. Hu, L. Xu, A. Zhang. (2012).Adaptive back-stepping trajectory tracking control of robot manipulator, J. Franklin Inst. 349 (3) (2012) 1087-1105.

[9] M.L. Corradini, V. Fossi, A. Giantomassi, et al. (2012). Discrete time sliding mode control of robotic manipulators: development and experimental validation, Control Eng. Pract. 20 (8) (2012) 816-822. 